Revue de l'Institut des langues et cultures d'Europe,

Amérique, Afrique, Asie et Australie

12 | 2010

La FASP : dix ans après...

\title{
Transplanted Man de Sanjay Nigam, une FASP Santé à visée transdisciplinaire et transculturelle
}

\author{
Marie-Lise Assier
}

\section{(2) OpenEdition}

\section{Journals}

Édition électronique

URL : https://journals.openedition.org/ilcea/444

ISSN : 2101-0609

Éditeur

UGA Éditions/Université Grenoble Alpes

Édition imprimée

ISBN : 978-2-84310-180-9

ISSN : 1639-6073

Référence électronique

Marie-Lise Assier, «Transplanted Man de Sanjay Nigam, une FASP Santé à visée transdisciplinaire et transculturelle », ILCEA [En ligne], 12 | 2010, mis en ligne le 20 mars 2023, consulté le 20 mars 2023. URL : http://journals.openedition.org/ilcea/444

Ce document a été généré automatiquement le 20 mars 2023.

Tous droits réservés 


\section{Transplanted Man de Sanjay Nigam, uneFASP SantĖ $\ddagger$ visĖe transdisciplinaire et transculturelle}

Marie-Lise Assier

\section{Introduction : choix de la FASP et objectifs didactiques}

Dix ans aprËs que Michel Petit a dĖfini la Fiction ¥ Substrat Professionnel comme une ' autre voie díaccËs ₹ líanglais de spĖcialisation a 1 , ce nouveau genre semble avoir honorÈ ses promesses, comme en attestent les nombreux articles en didactique des langues de spËcialitĖ Si, comme il le suggËre actuellement, la FASP change depuis quelques annĖes de visage et perd de plus en plus son cùtÈ ' substrat professionnel a pour dĖvelopper les aspects thriller, alors peut-Í tre faudrat-il chercher le ' profil FASP $\underline{a}$ chez des auteurs comme Sanjay Nigam. Ses Ėcrits, aux ambitions littĖraires, síappuient sur les expĖriences singuliëres du professionnel de la santĖ quíil est. Son roman, Transplanted Man², rËpond tout $\ddagger$ fait aux objectifs et aux besoins de líanglais de spĖcial itĖ dans le domaine SantÈ/ MĖdecine.

1 NĖ en Inde, Sanjay Nigama grandi en Arizona et a vĖcu dans divers lieux des . tats-Unis. II a travaillÈ avec G, nter Blobel, un laurĖat du Nobel Physiologie/ MĖdecine en 1999. II Écrit la nuit et consacre ses journĖes ‡ la recherche, notamment sur la formation et la rËgÈnĖration des organes. II est $\neq$ la tí te díun laboratoire de quinze chercheurs spĖcialistes du rein ₹ líuniversitÈ de Californie de San Diego. Líon sent poindre la fascination quíil a pour cet organe dans lí|̇oge quíil en fait dans Transplanted Man:

The kidney is full of wonders! Consider the glomurelus. What a beautiful structure, more beautiful than a sunflower! It filters nearly two hundred liters of plasma every day through its delicately fenestrated capillaries. Then there are the tubules, a million of them finely compressed together, as though fashioned by an emperorís goldsmith. Have you seen Fabergì's eggs, the very best of them? ImagineFabergÈstricken by dbsessivemania, creating thousands upon thousands of convoluted tubes, thin, intricatestructures, tightly packed, all 
dedicated to the supreme function of acid-base and edectrdyte balance ó so the lungs can breathe, so the heart can beat, so the brain can think! That marvellous organ is thekidney! (TM, p. 17)

2 Cet extrait illustre líidEe centrale de Sanjay Nigam sur les parties et le tout. Professeur de pÈdiatrie, mÉdecine, mĖdecine molĖculaire et cellulaire, il pense que la mĖdecine, la science et líart ont en commun le fait de rassembler des morceaux Ėpars pour en faire un 'rËcit a : ' He weaves parts into a whole ${ }^{\underline{a}}$. CUest ce caractËre ' trans $\underline{a}$ qui nous a immĖdiatement sÉduite chez líauteur, transculturel et transdisciplinaire, dans sa personnalitĖ, son ú uvre et son souci de crËer une unitÈ des I tres et des choses dans des liens parfois invisibles. Transplanted Man a ĖtÈ choisi comme Yearís Best Book par Publisherís Weekly, en 2002. II a reÁu de nombreuses critiques positives de revues et de journaux comme le Washington Post, le Utne Reader pour qui Sanjay Nigam est líun des dix romanciers qui changent la face de la fiction. Ce roman est traduit en plusieurs langues dont le franÁais sous le titre de Líhomme greffĖ Dans Transplanted Man, Sanjay Nigam donne ₹ voir diffÈrentes strates professionnelles ‡ líintĖrieur díun hüpital situĖ dans ' Little India a , ‡ New York, dans le quartier du Queens composÈ de multiples groupes ethniques issus díAsie. Les interactions entre langue et culture, entre reprËsentations et rĖalitĖ y apparaissent de faÁon magistrale. Les rapports au corps, aux croyances, au mystËre du sens de la vie et de la mort sont apprËhendĖs de diverses maniËres par les mËdecins et les patients sel on leurs origines. La richesse de ce roman, qui dĖpasse son fort substrat professionnel au profit de questions díordre Ėthique et philosophique, autorise une diversitĖ díapproches pĖdagogiques. Lí effet papillon $\underline{a} 4$, une des mËtaphores du roman, illustre le fait que bien des projets, conditionnËs par des croyances culturelles, sont souvent contrariĖs. Díabord immiscĖe dans la FASP pour comprendre líEvvolution du genre vers son propre dĖpassement, nous nous sommes intĖressĖe $\neq$ ce quíelle transmet, en privilÈgiant líobservation pratique.

3 En partant díextraits du roman, líun des objectifs didactiques est de rendre lítiudiant conscient des liens langue/culture et des rapports culture/comportements professionnels en síappuyant sur la mettaphore que constitue le personnage central, Transplanted Man. La langue du roman est $\ddagger$ son image: amalgame de fragments transplantĖs. Pour les Ėtudiants, ne plus avoir $¥$ affronter le bloc monolithique díun anglais quíls imaginent pur, parfait et uniforme síavËre rassurant et leur redonne confiance. La langue de spËcialitĖ est ici $¥$ saisir dans ses altèrations dues aux phÈnomËnes de mondialisation. DĖvelopper un regard critique sur les pratiques professionnelles constitue une autre dimension non nËgligeable de líttude de la FASP. La sĖquence pĖdagogique conduite auprËE díitudiants en Master Professionnel SantÈ, confirme que la FASP est un support idĖal pour la crĖation de scĖnarios, pour des discussions en cours, pour la mise en cause de schĖmas de fonctionnement ou de reprËsentations. Les Ėtudiants śímpliquent activement et se libËrent de la peur de faire des erreurs. -gĖs de $22 \ddagger 60$ ans, certains ont arrí tĖ toute Ėtude depuis vingt ans. Cette nouvelle accessibilitÈ de la langue et de son apprentissage les conduit bien souvent ₹ vouloir prolonger líexpĖrience au-del $\neq$ des 24 heures accordĖes par module et beaucoup $\mathrm{dËsirent}$ continuer $\neq$ lire $\neq \mathrm{f}$ pratiquer. 


\section{Transplantation rÈussie díune FASP en cours de langue de spĖcialitĖ}

La mĖdiation didactique quíimplique une fiction ¥ substrat professionnel porte, díune part, sur la vËrification de la fiabilitĖ des donnĖes, et díautre part, sur la ' rĖcupÈration a pĖdagogique díun matĖriau essentiel permettant de construire des connaissances utiles pour analyser líancrage culturel et contextualiser les apports langagiers. Lítide díune FASP peut síavĖrer Í tre un excellent moyen pour qui veut attĖnuer les dĖcal ages ou les clivages entre enseignement secondaire et enseignement universitaire et entreSciences et Littèrature.

4 Dans sa transversalitè, le roman de Sanjay Nigam fait passer le lecteur díun point de vue $\ddagger$ un autre, díune logique $\ddagger$ une autre, díun lieu $\ddagger$ un autre. La volontĖ de transgresser la nature, les lois existentielles, les codes, les conventions, y compris dans le domaine mĖdical, conduit $\ddagger$ une certaine forme de transcendance chez des personnages communs qui veulent simplement vivre un peu mieux ou aider $\ddagger$ mieux vivre. Le titre est metaphorique tout comme le contenu du roman qui force $\ddagger$ une lecture transitionnelle, entre rËalitÈ et reprËsentations. La transplantation, bien quíorganique, est ‡ líimage de ce qui se passe dans le minĖral ou le vËgĖal, tous rËgnes confondus.

He nodded and again stared out of the window at the willow tree He had always liked looking at trees: If he were a philosopher, or a scientist lucky enough to study a purely abstract question for its own sake, héd try to formulatea theory of trees. Not just thekind in his backyard but also the ductal trees of lungs and kidneys, and countless other tredike structures: corals, blood vessels, lightning, river deltasö Might trees harbor a great secret that transcended theanimateand inanimate? (TM, p. 243)

5 Le mystëre de la vie dans líanimĖ et dans líinanimÈ fait líobjet de spĖculations diverses selon la discipline quíon Ėtudie, selon la culture ł laquelle on appartient. Cette lecture ne peut conduire quíf plus de tolÈrance et de curiositĖ. Les expĖriences des personnages síinscrivent dans un brassage de cultures ethniques, dans des systËmes de pensĖe aussi ÈloignĖs que ceux de lílnde et des .tats-Unis et dans un milieu trËs spĖcifique, celui de líhùpital. La fá́on dont les individus apprènendent le temps, líespace, líorganisation sociale et professionnelle nous ai dent ₹ dĖconstruire les visions stĖĖotypĖes que nous en avons. Cette nouvelle image du milieu hospitalier, du statut de la santĖ, autant physique que mentale, au sein díune socièṫ̀ plurielle, ai de $\ddagger$ adopter des comportements communicatifs plus souples. Sanjay Nigam semble Ėgalement vouloir pacifier líopposition entre des sensibilitĖs ' occidentales $\underline{a}$ et 'orientales $\underline{a}$ dans le domaine de la santĖ/ mÈdecine, caricaturĖe et incarnĖe dans un autre domaine par Tiger, chef cuisinier, qui compose ses plats entre deux mondes: celui du go ${ }^{\circ} \mathrm{t}$ fade et celui des Èpices.

6 Le roman de Sanjay Nigam, par sa richesse disciplinaire et culturelle intÈresse particuliërement les lecteurs que sont nos Ėtudiants de Master professionnel en langue de spĖcialitĖ Dans le domaine de la santĖ notamment, leurs parcours sont hÈtĖroclites: issus díEtudes de biologie, chimie, sociologie, psychologie, droit, ou sortis díĖcoles díinfirmiËres ou díinstituts divers, certains travaillent, díautres ont perdu leur emploi, díautres encore poursuivent un cursus dit 'normal a . Certains níont jamais voyagè, díautres ont participĖ plusieurs fois $\ddagger$ des actions humanitaires $\ddagger$ diffËrents endroits du 
globe. Ils viennent de divers milieux socio-culturels et de pays variÈs (Pakistan, Inde, Afrique, Angleterre, etc.).

\section{VisÈe transdisciplinaire de Transplanted Man}

Dans le roman, la figure du chercheur se modifie au grÈ des contextes. Son identitĖ change selon qui la perÁbit. Le Dr Ranjan pense que ' two decades of failure- font de lui un ratĖ (TM, p. 90). Cependant quand il parle de ses recherches avec le Dr Menaka Bushan son discours contient des accents enthousiastes. En revanche, ses collËgues le dĖnigrent jusquíau jour o־, par chance, il trouve la molĖcule contre líinsomnie. La direction de líhùpital, quant $\ddagger$ elle, níenvisage le chercheur et sa recherche que sous líangle du profit et de la rentabilitè. II síagit, dans toutes ces situations, du mí me homme, díune mí me fonction qui pourtant, pris dans des niveaux diffÈrents díune mí me rĖalitè, ne va pas utiliser et entendre le mí me langage. Le milieu professionnel en tant que locus est dĖpeint dans le roman de faÁon trËs rËal iste, et comprend la salle díopËration, les urgences, la sal le de rĖanimation mais aussi les couloirs, les ascenseurs, les chambres, les halls. Ĺhùpital ressemble $\ddagger$ une entreprise qui doit faire du rendement et afficher de bons rËsultats. Le laboratoire de recherche doit trouver des produits permettant de financer les autres services. Le personnel est soumis $\ddagger$ une hiËrarchie trËs pesante, fortement ressentie par Sonny, le docteur central du roman. Outre les descriptions trËs prËcises de certaines opËrations, expËriences, traitements et notes de mĖdecin, le thËme de la transplantation donne lieu ₹ diverses mĖtaphores. Líh Ùpital devient alors une machine de guerre o les conditions de travail et la frËnĖsie hospitaliËre emportent les personnages malgrĖ eux loin de leur vie propre. Ils ont du mal ₹ se connađ́re. Ils sont en perte et en quí te díidĖaux, de rí ves. Leurs Ėmotions fragmentĖes doivent síadapter ₹ leurs environnements, ce qui finit par les sĖparer díeux-mí mes. Dans ce qui semble plutùt Í tre un chaos gĖnĖral, le lecteur glisse dans certaines pathologies et comprend leur fonctionnement de líintÈrieur. Ainsi, gr, ce ₹ líinertie et la vision ralentie de líhomme hypocinĖtique, observe-t-il les mouvements dĖmentiels díune sociètÈ qui tourne $\ddagger$ vide. Les petits dĖtails restituĖs de faÁon amplifièe par un cerveau qui fragmente au lieu de synthÈtiser les informations reÁues ne permettent pas díaccĖder au sens global des phÈnomËnes perÁus. Sanjay Nigam montre dans sa fiction les liens qui existent entre des conditions de travail et des comportements.

Le substrat professionnel de la FASP alimente la connaissance de líanglais de spĖcial itĖ qui fait partie díun plus grand ensemble langagier et qui donne lieu ₹ líapprentissage communicationnel et actionnel. Tom Hutchinson rËsume bien la position de líESP dans sa prËsentation en forme díarbre: The Tre of 且T (Hutchinson, 1987, p. 17). Dans líaspect spĖcifique díun domaine comme celui de la santĖ/ mĖdecine, la langue de spËcialitÈ est $\ddagger$ inclure dans une transversalitĖ disciplinaire directement liĖe $¥$ la culture comportementale. Florence Mourlhon explique que le vocabulaire spĖcialisÈ est sous-tendu par un mode de raisonnement et des logiques de fonctionnement: le ' discours (est) contraint par une situation díĖnonciation (lieu social professionnel) † visĖe pragmatique (Mourlhon, 2008, p. 36). Nombre díactivitĖs en langue de spĖcialitĖ font effectivement appel $\ddagger$ des compètences dĖcloisonnĖes comme rËdiger un compte rendu de lettres, prendre des notes, etc. Les projets prËsentĖs par les Ėtudiants doivent Í tre crĖdibles et authentiques autant que possible. Ils śínsËrent dans des pratiques utiles pour leur discipline majeure. Ils font cependant appel $\ddagger$ des savoir-faire et $\ddagger$ des 
savoir-í tre transversaux. Or, le roman de Sanjay Nigam propose des modت̈es de ce type.

8 La neuropÈdagogie a montrÈ líimportance des rÈseaux de sens dans líapprentissage et líimportance de líaffect ${ }^{5}$. De Varela dĖcrit, dans Lílnscription corporelle de líesprit (Varela, 1999, p. 134), ' la rËgle de Hebb a qui donne scientifiquement les raisons díessayer díEtablir le plus de liens possibles, de connexions et díenrichir les rËseaux:

Donald Hebb suggìra que líapprentissage peut reposer sur des changements qui, ‡ líintĖrieur du cerveau, dĖcoulent du degrÈ de corrèlation de líactivitĖ des neurones: si deux neurones ont tendance ₹ I tre activĖs simultanÈment, leur connexion est renforcĖe; dans le cas contraire, la force de la connexion est diminuĖe.

Ces propos confirment que la langue de spĖcialitĖ doit Í tre apprènendĖe dans ses interfËrences avec la langue gÈnÈrale et dans des situations contextualisĖes, ce que donne ₹ voir le roman qui est notre objet díEtude. Líouverture sur díautres faÁons de dire et de fair re permet díEtablir des Ėtudes comparatives de systËmes.

Pour Besarab Nicolescu, physicien et thÈoricien au CNRS, la transdisciplinaritÈ est une nouvelle approche scientifique, culturelle, spirituelle et sociale nÈcessaire ‡ líavancĖe des sciences. Dans La transdisciplinaritĖ, Manifeste, il voit le langage disciplinaire comme ' barrage apparemment infranchissable pour un nËophyte a (Nicolescu, 1996, p. 63). II retrace le besoin de liens entre les diffĖrentes disciplines qui síest fait sentir vers le milieu du vingtiËme siëcle avec lí́mergence de la pluridisciplinaritĖ et de líinterdisciplinaritè ce qui le conduit $¥$ dÈfinir les diffĖrents termes: 'La pluridisaiplinaritÈ concerne lítiude díun dojet díune seule et mí me disaipline par plusieurs disciplines ₹ la fois [Ö] Autrement dit, la dÈmarche pluridisciplinaire dĖborde les disciplines mais sa finalitÈ reste inscrite dans le cadre de la recherche disciplinairea (I bid., p. 64-65)6. La langue de spĖcialitĖ est pluridisciplinaire puisquíelle engl obe diffĖrents domaines, diffèrentes langues, mais ses buts restent inscrits dans son cadre LANSAD?. MÈthodologiquement, elle peut sínspirer díautres disciplines. La didactique des langues puise dans les dÈcouvertes faites en neurobiologie ou en neurosciences pour mieux comprendre les fonctionnements sous-jacents aux actes langagiers et mettre en place de nouvelles approches, essayer de nouveaux outils. ' LinterdisciplinaritĖ a une ambition diffèrente de celle de la pluridisciplinaritĖ Ele concerne le transfert des mèthodes díune discipline ₹ líautre [Ö] sa finalitĖ reste aussi inscrite dans la recherche disciplinairea (Ibid., p. 65).

10 Plus enrichissante encore est la transdisciplinaritÈ dont Besarab Nicolescu prÙne les mĖrites: ' La transdisciplinaritĖ concerne, comme le prËfixe ' trans a líindique, ce qui est $\ddagger$ la fois entreles disciplines, $¥$ travers les diffèrentes disciplines et au-deł de toute discipline. Sa finalitĖ est la comprÈhension du monde prËsent, dont un des impËratifs est líunitÈ de connaissance ${ }^{a}$ (Ibid., p. 66-67). Líanglais de spĖcialitĖ a une vocation transdisciplinaire car son objet díEtude, la langue, síadapte ₹ díautres disciplines et faÁonne une discipline entre les sciences et les langues. Cette langue est spĖcifique mais a recours ¥ une langue situĖe au-delł de toute spÉcificitÈ et dont les Ėvolutions constantes obligent $\ddagger$ repenser les apports: [de] quelle(s) langue(s) parlons-nous? Síagit-il díun anglais national, international ? Elle doit parfois I tre trËs prËcise et dans díautres cas, se satisfaire díapproximations. Dans le milieu mĖdical, la terminologie exacte est parfois remplacĖe par des termes plus gÈnÈraux, voire des mÈtaphores. La langue de spĖcialitĖ se conjugue avec une langue nommĖe ' langue vÈhiculaire ${ }^{a}$ ou

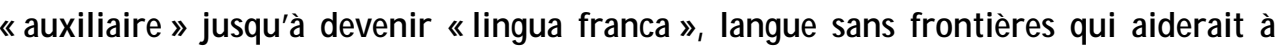
'transcender les cultures a (Hay, 2009, p. 64). Pour Besarab Nicolescu, il níy a pas 
quíune rĖalitĖ; il y a plusieurs niveaux de rÈalitÈ parfois en un mí me lieu, en un mí me moment: ' La structure discontinue des niveaux de RËalitĖ dËermine la structure discontinue de líespace transdisciplinaire [Ö] la transdisciplinaritĖ síntÈresse ‡ la dynamique engendrËe par líaction de plusieurs niveaux de RĖalitĖł la fás a (Nicolescu, 1996, p. 66). Ces rĖalitĖs interagissent entre elles comme le dÈmontre Sanjay Nigam dans son roman.

11 La FASP est une voie intĖressante pour la didactique des langues de spĖcialitÈ car elle montre la plasticitè du langage qui se modifie au grÈ des situations. Ces altèrations sont soulignĖes par Sanjay Nigam dans une dimension transculturelle. II donne $\ddagger$ voir ces glissements de sens dans des processus plutùt que dans des faits apparents et achevĖs. Les Ėtudiants apprĖcient la rencontre impromptue avec des personnages vraisemblables, bien que parfois caricaturaux, qui correspondent $\ddagger$ leur univers et auxquels ils peuvent sídentifier.

\section{VisÈe transculturelle de Transplanted Man}

Dans Transplanted Man, du fait que líhùpital est majoritairement peuplè dítrangers, cÙtÈ soignants, cùtÈ soignÈs, les langues dĖteignent parfois les unes sur les autres et líanglais se mí le au hindi :

ì Sonali, I love you! I love you exactly as you areî She squinted at him ì Achanack ye mohabat hakan say agayee?i ì You donít understandî he said. ì People have warped your mind.î For the first timein weoks she spoketo himin English. ì Excuseme, whose mind did you say is warped?i (TM, p. 100)

Quand les mots ne sont plus fiables ou quand ils deviennent non compris, la mĖtaphore vient pallier le manque sĖmantique. Dans le roman, ce processus est exagËrÈ par líutilisation díallĖgories. Ainsi, le psychologue Docteur Gri devient-il Guruji, gourou, par adaptation au quartier dont les habitants ne savent pas ce quíest un psychiatre ou un psychologue.

Líaspect culturel est dÈterminant car la langue est au cú ur des reprÈsentations que nous avons de la rÈalitĖ Ele les modÈlise, les vËhicule. Suite ₹ de nombreux autres auteurs, Sophie Moirand síintĖresse ₹ la composante socioculturelle de la compĖtence de communication. Ele la dËcrit comme la connaissance et líappropriation des rËgles sociales et des normes díinteraction entre les individus et les institutions, la connaissance de líhistoire culturelle et des relations entre les objets sociaux. Elle la dĖfinit $\ddagger$ la suite de composantes plus connues: composante linguistique, discursive, rÈfĖrentielle (Moirand, 1982, p. 20). ¿ ces composantes, síajoute dans le Cadre Commun de RËfËrences des Langues, la perspective actionnelle qui met en avant líexĖcution des $\mathrm{t}$, ches en langue cible.

13 Le concept 'isma du roman est un bon exemple de više transdisciplinaire et transculturelle dans une approche actionnelle. Il constitue le thËme central du seiziËme chapitre intitulÈ 'The Pose for the Ismª. Deux adolescents siintĖressent $\ddagger$ líhomme hypocinÈtique, par jeu au dĖpart, par intĖrít ensuite, et pour líun deux, par empathie plus tard. Jay, le plus $m^{\circ} \mathrm{r}$ des deux, pense que ce serait bien quíil y ait un 'isma pour chaque individu: 'An ism that has nothing to do with another ism but thatís the same whether youíre brown or white or grean $\underline{\text { a }}$ (TM, p. 166). Cet ' isma aurait le pouvoir díarrí ter tout conflit. En voyant líhomme hypocinètique, Atul, son copain, pense quíil pourrait incarner ce concept par une pose: ' If wecould find theright pose the posefor theism maybeeverybody would do it and peoplewould stop fighting! a (Ibid.). J Jay en doute. Cependant, líhomme hypocinĖtique garde la pose que les adolescents lui ont fait 
prendre et qui ressemble Ėrangement $\ddagger$ celle du penseur de Rodin. Líhomme hypocinĖtique attire le regard des passants intriguĖs par la posture qui viennent en nombre et qui finissent par payer pour ce phĖnomËne Ėtrange. Les enfants síenrichissent. Le 'isma a est dĖvoyÈ par líargent. Atul, quelques temps plus tard, prendra conscience de son erreur : ' Henolonger believed in the posefor-theism In fact, he was amazed that hehad been sonaive (TM, p. 315). Ce concept, figÈ en pose, nemettrait-il pas en garde contre toute forme de dogmatisme?

Transplanted Man constitue une figure qui rÈpond partiellement $\ddagger$ cettequestion : il est líallĖgorie de lílnde, corps mosaQ̂ue (TM, p. 70-76). Ce surnom a Ėtè donnĖ au personnage principal car il a subi des greffes sur sept de ses organes vitaux. Son corps composite est toujours prí $t \neq l$, cher $\ddagger$ un endroit ou un autre car les divers fragments, provenant de donneurs díorigines et de religions diffÈrentes, semblent incompatibles. Ministre de la santĖ en Inde, Transplanted Man reprËsente celui qui peut rĖunir les diffËrentes communautĖs de son pays: ' Peoplebegan to say hewas thelast of great leaders, theonly one left who could mobilize a fractious nation and chart its future - (TM, p. 11). II est fier de sa nation et la comprend díautant mieux quíil lui semble en Í tre líincarnation : ' [Ö] hereflected, all his suffering had given himinsight intothemaladies of thecountry a (TM, p. 12). Pourtant líhùpital new-yorkais est son dernier refuge car sa vie est menacĖe de líintĖrieur (rejets des greffes) et de líextÈrieur (attentat politique). Sonny, docteur en charge de ce patient, fait tout pour colmater les brËches, recoller les morceaux, changer les piëces, en rajouter si nĖcessaire, mais il síEpuise $\ddagger$ cetravail difficile qui fait Ėcho ¥ sa propre vie. LíhÙpital, dans le quartier de Little India ¥ NewYork, est un microcosme des USA avec en arriËre plan lílnde et sa vie politique. Le lieu, bien que dĖpeint de faÁon rĖal iste, semble avoir une vie propre rËvèĖe par les mĖtaphores qui le dËcrivent: ' battle war a , ' army ${ }^{\text {a }}$, ' hospital choreography ${ }^{a}$. Les personnages qui gravitent dans et autour de cet ensemble sont tous, en quelque sorte, des ' transplantĖs a venus de diverses rËgions du globe. Ils vivent plus ou moins bien leur transplantation ; beaucoup dÈveloppent des mal adies physiques ou mentales. Le corps a des liens Ėvidents avec la culture et avec son environnement. Passer díune culture ₹ une autrelíaffecte:

ì These days the main show seems to beheart diseaseî said Sonny. Ì At threethis morning I was paged to the $R$ to see a forty-four-year dd man with severechest pain. That makes five Indian men in their forties admitted this week with heart attacks. Itís an epidemic. Scary.î Dr Ranjanís face becamegrim ì Even scarier if youírean Indian man in your forties. Just last month the father of a girl in my daughterís Bharatnatyam dass died of a heart attack. One day he was at the peak of his careør, the next day a six-year-dd was fatherless.i ì l canít figureit aut,î said Sonny ì Therés got to bemoreto it than samosas.î i The real reason must bea mismatch between our genes and theenvironment,î said Dr Ranjan. ì this environment. We didnít evdve for this lifestyle Our clocks tick to a different rhythmî Sonny nodded sowly. (TM, p. 151)

Le corps, les rapports au corps, les comportements sont ancrÈs dans une culture. II semble essentiel díaborder ces questions avec des Ėudiants, futurs professionnels de la santÈ Le passage suivant est le dËveloppement díun thËme prËsent dËs le dĖbut du roman :

ì Whatís thestory? ì This guy camein with no pulse Wéve been resuscitating for fiveand a half minutes, but nothing ó nothing at all. [Ö] This man seems awful young for a heart attack.i ì Thatís what we get for coming hereî interjected an old nurse from Gryana. ì We leaveour own land, work hard in someoneedsés, and dieyoung.î (TM, p. 22)

Ces idĖes sur les interfËrences entre líhomme et son milieu font Ėcho $\ddagger$ des recherches actuelles comme en attestent les travaux de Boris Cyrulnik, Edgar Morin, Francisco 







